Like many malignancies, the development of colorectal carcinoma (CRC) can be considered as an imbalance between the compromised process of programmed cell death (apoptosis) and excessive, uncontrolled proliferation. Several mutations and epigenetic alterations are acquired during colorectal carcinogenesis. These are responsible for the cell cycle regulation, cellular sensitivity to pro- and antiapoptotic factors, cell proliferation, angiogenesis, invasiveness, as well as metastatic potential. The molecular alterations, along with their morphological expressions, have been recognised in detail, and most of the CRC cases can be attributed to either adenoma-carcinoma or serrated neoplasia pathways: in the first, the antiapoptotic features prevail; while in the second, the proliferative activity is of the utmost importance. The aim of the work is to discuss the influence of selected drugs and dietary compounds on the proliferation and apoptosis in CRC

Key words: colorectal carcinoma, apoptosis, proliferation, drugs, dietary compounds.

Contemp Oncol (Pozn) 2014; 18 (4): 222-226 DOI: $10.5114 /$ wo. 2014.44296

\section{The effects of selected drugs and dietary compounds on proliferation and apoptosis in colorectal carcinoma}

\author{
Miroslaw Kiedrowski ${ }^{1,2}$, Andrzej Mroz $^{3}$ \\ ${ }^{1}$ Faculty of Human Nutrition and Consumer Sciences, Warsaw University of Life Sciences \\ - SGGW, Warsaw, Poland \\ ${ }^{2}$ Department of Pathology, Maria Sklodowska-Curie Memorial Cancer Center and \\ Institute of Oncology, Warsaw, Poland \\ ${ }^{3}$ Department of Gastroenterology and Hepatology, Medical Centre for Postgraduate \\ Education, Warsaw, Poland
}

\section{Introduction}

Most colorectal cancers (CRC) develop as a result of a long-term process of acquiring mutations and epigenetic alterations [1, 2]. Several dozen mutations have been recognised thus far, most of them being categorised, along with their morphological expression, in two carcinogenetic pathways: classical adenoma-carcinoma and serrated neoplasia sequences. However, all pathways result in the selection of neoplastic cell clones, gaining some essential features of malignancy, like apoptosis resistance or uncontrolled proliferation. Many drugs are known to influence the apoptosis and proliferation. The cytostatic agents are strong but not very specific inducers of apoptosis and/or inhibitors of proliferation. On the other hand, many non-cytostatic drugs can influence proliferation or apoptosis in the normal and transformed colonic mucosa. Additionally, food is a known source of numerous active compounds, but the practical application of this finding has been extremely limited thus far [3]. It is expected that such activities could support novel strategies of colorectal cancer prevention and therapy, but this would require properly designed prospective studies. The aim of the work is to discuss the impact of selected drugs, as well as certain bioactive food ingredients, on apoptosis and proliferation in colorectal cancer cells.

\section{Apoptosis and proliferation in colorectal cancer}

Apoptosis is usually defined as a caspase-dependent programmed cell death, and it plays a key role in all stages of ontogenesis. The resistance to pro-apoptotic factors has been reported in many malignancies. Proliferation is also strictly regulated. As in apoptosis, both stimulating and inhibiting factors regulate proliferation; therefore, any carcinogenetic process can be considered as an imbalance between apoptosis and proliferation, with all applicable restrictions. The phenotype of the cancer cells may change, and new biological features are often acquired over time. Some of them can be clinically significant, like the radioresistance and chemoresistance. Many factors may be involved in the colorectal carcinogenesis, like $\mathrm{Bcl}-2$ family members, P53, P21, and many others [4, 5].

A relationship between apoptosis, the mitotic index, and the surveillance has been shown in colorectal carcinoma [6]. Tumour cells often show overexpression of anti-apoptotic proteins. They also accumulate mutations in the tumour suppressor genes, as well as the protein kinase genes, the products of which are engaged in the regulation of proliferation [7]. They may also 
display a lack of specific molecules acting as apoptosis inducers. It is believed that P53 gene products, as well as BCl-2 family and C-myc genes, act as important apoptotic regulators in colorectal carcinogenesis. Many other genes and proteins are also involved in this process. Their phenotypic expression reflects the changes in the molecular machinery of transformed cells, and manifests as certain morphological patterns in light microscopy and in the clinical course of the disease [8].

Considering factors affecting apoptosis and the proliferation processes, the clinical significance of such intervention should be considered also with non-oncologic drugs and dietary approaches. In the present state of knowledge, the mutated genotype of colorectal cancer cells cannot be restored to its initial state, so most medical interventions tend to eliminate the transformed cells by the induction of apoptosis and/or other forms of cell elimination. Both chemotherapy and radiotherapy act in that way, leading to apoptosis with the effector caspase activation and/or necrosis.

\section{The effect of selected pharmacological agents and dietary compounds on proliferation and apoptosis in colorectal cancer}

There are several reports regarding the impact of pharmacological interventions on colorectal carcinogenesis. Only some authors discuss the proliferative activity and apoptotic susceptibility/resistance in the colonic mucosa, as well as the underlying molecular mechanisms. Several categories of pharmacological agents are known to affect these phenomena, and these include non-steroidal antiinflammatory drugs (NSAIDs), histamine receptor $2(\mathrm{H} 2)$ antagonists, HMG-CoA reductase inhibitors (statins), unsaturated fatty acids, carnitine, vitamin D, and calcium [9].

The NSAIDs are the only group in which chemopreventive actions in the colonic mucosa were unequivocally proven. Several randomised studies showed that sulindac, celecoxib, and aspirin inhibit the growth of adenomas in the colon, and in patients with familial adenomatous polyposis they are even able to cause regression of existing lesions [10-12]. Blocking the cyclooxygenase 2 increases apoptosis and inhibits the proliferation and angiogenesis in the adenomas. Some authors suggest that the inflammation could play an even more considerable role in the serrated neoplasia pathway than in classical adenoma-carcinoma sequence [13]. However, the anti-inflammatory mechanism seems insufficient to display all effects. Hanif et al. demonstrated that treatment of HT-15 and HT-29 cells with PGE2, PGF2 alpha, and PGI2 following a variety of protocols, and at different concentrations failed to reverse the effects of NSAIDs on proliferation, apoptosis, and cell cycle phase distribution. Therefore, prostaglandin-independent mechanisms have been postulated [14].

It is worth noting that COX-2 expression appears in a substantial number of benign epithelial polyps as well as CRCs. In the CRC the expression of COX-2 was reported to alter cohesiveness and apoptotic resistance, thus accelerating tumour growth and infiltration [15].
Classical NSAIDS inhibit the COX-2 activity to a limited extent, while the chemopreventive properties of aspirin have been shown even in the small, "cardiac" doses of acetylsalicylic acid.

Several investigators demonstrated that COX-2 inhibitors sensitise cells to the extrinsic-induced apoptosis. The pro-apoptotic effect of Fas ligand (FasL) binding was potentiated regardless of the COX-2 inhibition. Reinacher-Schick et al. have studied the impact of mesalazine (5-aminosalicylic acid) on apoptosis and proliferation in colonic mucosa of patients with sporadic colonic polyps. They showed a statistically significant increase in the incidence of apoptosis as early as 1-3 days after starting treatment. This effect persisted during the study course. In the mesalazine-treated group a lower proliferation of enterocytes was shown, when compared to the control [16]. In another study the statistically significant selectivity of mesalazine was demonstrated. The drug induced the apoptosis in the cancer tissue, but the adjacent mucosa remained intact [17]. Mesalazine - a veteran drug in inflammatory bowel diseases seems to inhibit the proliferation of cancerous cells as well. Reinacher-Schick et al. compared the effect of mesalazine with some cytotoxic drugs of a well-established position. They demonstrated that mesalazine, albeit less effectively than sulindac and indomethacin, limited the proliferation of cancer cells, stopping the cell cycle at the mitosis stage. The drug in no way affected the microtubules polymerisation. The tested concentrations corresponded to those obtained during the standard therapy. The authors have also proven that the apoptosis activation in the colon cancer cells was most likely due to the caspase 3 activation, although the activity of $\mathrm{Bcl}-2$ family proteins was not affected [18]. Such a mechanism was not previously taken into account in relation to NSAIDs.

Another classical NSAID, sulindac, inhibited the proliferation and increased the apoptosis in the study of two colon cancer cell lines - RKO and HT-29. Its effect was accompanied by an increase in the activity of 15-lypoxygenase-1 (15-LOX-1), which is the key enzyme metabolising the intestinal linolenic acid to the 13S-hydroxyoctadecadienoic acid (13(S)-HODE) - an apoptosis inducer [19].

In the clinical trials the sulindac administration reduced the size and number of colorectal polyps in patients with familial adenomatous polyposis. No reduction in the proliferative activity was shown, but only an increase in the apoptotic activity in the colonic mucosa.

With regard to another classical NSAID - indomethacin - the inductive activity has been shown with reference to the expression of $\beta$ - and $\gamma$-catenin, as well as the T-cell factor (TCF) [20]. $\beta$-catenin plays an important role in the so-called WNT pathway, and its expression is often dysregulated early in the course of carcinogenesis.

$\mathrm{H}$ 2-receptor inhibitor - cimetidine - seems to improve the prognosis in CRC, but the exact mechanism of this activity is unknown. Plausible mechanisms may include blocking the cell proliferation and inhibition of the suppressor $T$ lymphocytes, but the increase in apoptosis of cancer cells cannot be also ruled out [21, 22]. Some authors investigated the in vitro effect of histamine, cimetidine, and ranitidine on human colon cancer cell lines - Caco2 and 
LoVo. Histamine did not influence apoptosis or proliferation in any of these lines, but ranitidine caused a reduction in proliferative fraction in the $\mathrm{Caco} 2$, both alone and in the presence of histamine, whereas cimetidine restricted the proliferation, but only in the co-presence of histamine. At the same time, $\mathrm{H}$ 2-receptor antagonists did not have any effect on the LoVo line proliferation. Both drugs activated apoptosis in the Caco-2, while ranitidine only in the LoVo line. The H2-receptor antagonists may affect the proliferation and apoptosis in the histamine receptor independent mechanisms. It is likely that this action can be explained by the influence on the COX-2 synthesis route. Even though the conclusions from the studies performed on the isolated cell lines may not reflect the in vivo mechanisms, the anti-carcinogenic potential of histamine antagonists is still to be confirmed, especially in light of prospective clinical studies showing the immunomodulatory effect of the perioperative cimetidine administration in CRC [23].

Statins, dedicated mainly to primary and secondary prevention of cardiovascular events, are another group, and they are interesting due to their effect on the apoptosis and proliferation in CRC. Wachtershauser et al. demonstrated that mevastatin increases the anti-proliferative effect of butyric acid in the Caco-2 cell line. Moreover, it has also presented some pro-apoptotic activity [24]. There is also some data indicating the potentialisation of the sulindac pro-apoptotic effect by lovastatin [25].

Butyrate - a short-chain fatty acid - is a promising new therapy for irritable bowel syndrome (IBS) [26]. It should be noted that butyrates constitute a preferred energy source for colonic epithelial cells. Furthermore, butyric acid acts as an anti-proliferative factor and may promote the differentiation and maturation of the tumour cells. Mevastatin arrested the cell cycle at G1 phase after 24 hours, with the transition to the G2/M phases after 72 hours, which was accompanied by the suppression of cyclin-dependent cdk 4 and cdk6 kinases as well as cyclin D1. At the same time, increased activity of the P21 and P27 proteins - the cell cycle inhibitors - was noticed. Incubation with butyrate was shown to have a synergistic effect, which was easily reversible by the mevalonate addition (a short chain fatty acid), but not the intermediate metabolites of the cholesterol synthesis pathway. In light of these reports, statins are a promising complement to the anti-neoplastic therapy in CRC, acting by the increase of apoptotic activity and cell cycle arrest in the GO/G1 phase, i.e. the suppression of cancer cell proliferation.

Several other substances have been reported to display significant activity against CRC cells. Wenzel et al. demonstrated the pro-apoptotic effect of L-carnitine administered with palmitoylcarnitine, which was the effect of intensification of fatty acid oxidation [27]. In fact, the dysregulation in mitochondrial transport and $\beta$-oxidation is quite a common finding in the transformed cells. The effect was present only during simultaneous treatment with both compounds and it requires confirmation in vivo.

Colorectal cancer is considered to be a diet-dependent disease, but the only convincing dietary risk factors are red and processed meat and alcohol consumption [28]. The role of several other nutritional compounds in col- orectal carcinogenesis remains a matter of investigation. Vitamin D, calcium, fibre, unsaturated fatty acids, milk fat, and several natural plant substances have become a matter of debate. Under physiological conditions the proliferative activity of colonic epithelial cells remains in some relation to the food ingredients. The nature of such a relationship may be direct or indirect, namely hormone (gastrin)/growth factors (epithelial growth factor)/polyamines mediated. Some large observational studies showed the preventive role of dietary fibre consumption in regard to CRC [29]. Fibre is defined as a group of compounds that are the structural elements of plant cells and are resistant to digestion in the gastrointestinal tract. There is a limited amount of studies concerning the relationship between dietary fibre and cellular metabolism in regard to apoptosis and/or proliferation. There are Interesting reports on the impact of dietary fibre by sensitising cells to pro-apoptotic factors. The protective role of fibre was confirmed by a recent meta-analysis of twenty-five prospective studies, even though there are still a number of inconsistent results concerning the association between fibre intake and carcinogenesis [30]. Several mechanisms were proposed to explain the protective role of the dietary fibre, e.g. increased stool bulk, dilution of carcinogens, and reduced transit time through the gut. Oligosaccharides, which constitute a water-soluble fibre fraction - raffinose, lactulose, oligofructose, stachyose - are subject to bacterial fermentation to short-chain fatty acids. The outcome compounds are able to stimulate the proliferation of probiotic lactic fermentation bacteria (Lactobacilli and Bifidobacteria) that are believed to have some anti-carcinogenic properties [31]. A growing body of evidence shows that probiotic strains are able to inhibit initiation or progression of carcinogenesis. This ecologically diverse group of microorganisms displays a variety of actions, which - due to the large amount of mechanisms - are beyond the scope of this review. It is worth noting that several strains demonstrate a significant impact on apoptosis and proliferation in CRC - namely Lactobacillus reuteri (which has an impact on proliferation by affecting Cox-2 and cyclin D1, as well as cell survival - by Bcl-2, Bcl-xL-mediated pathways), L. acidophilus and L. casei (5-fluorouracil apoptosis induction), Bifidobacterium longum and L. acidophilus (antioxidative activity, inhibiting linoleic acid peroxidation), Streptococcus thermophilus (releasing reactive oxygen species protective factors), Pediococcus pentosaceus FP3, L. salivarius FP25/FP35 (adhere to CRC cells and trigger biosynthesis of short-chain fatty acids), and many others [32].

The secondary bile acids (deoxycholic and lithocholic) - derivatives from the primary bile acids, produced due to bacterial activity in the colon - increase the proliferation of enterocytes in a direct manner. An increase in the activity of kinase $C$ and in eicosanoids production was reported [33]. Eicosanoids are cellular signal transducers, activators of membrane lipids peroxidation, and inhibitors of apoptosis.

A preventive effect is attributed to the consumption of fruits and vegetables (rich in natural antioxidants), adequate intake of calcium and curcumin, which contains diferuloylmethane, the yellow pigment found in Indian saffron. This inhibits the cell cycle in G2/M, contrary to 
NSAIDs, which inhibit the G1 phase. Curcumin also exhibits a strong anti-proliferative activity in the CRC-derived cell lines, it reduces the cyclin-dependent kinase D and $E$ levels, and also induces apoptosis. Curcumin was shown to act as a chemosensitiser to a variety of chemotherapeutic agents (e.g. doxorubicin, 5-FU, vincristine, paclitaxel, melphalan, cisplatin), and in various cancers (CRC, breast, pancreas, gastric, liver, etc., as well as several haematological malignancies). Used for centuries in traditional herbal medicine and as a spice, it was shown to downregulate various regulatory pathways, genes for NF-kB, STAT3, COX2, growth factor receptors, antiapoptotic proteins, and multidrug-resistance proteins. Interestingly, curcumin was also shown to protect normal organs from chemo- and radiotherapy-induced toxicity, probably by inducing activation of NRF2 and antioxidant enzymes (e.g. glutathione peroxidase), in addition to its ability to quench free radicals [34].

In conclusion, proliferation and apoptosis in CRC should not be considered as isolated phenomena. Whole molecular pathogenesis, and systemic and clinical context should always be considered. Numerous pathogenetic pathways contribute to the development of the disease. We believe that molecular studies of apoptosis and proliferation in CRC may offer new horizons for potential pharmacological and dietary interventions. To take advantage of wellknown drugs and dietary modifications, some issues should be emphasised:

1. Several pharmacological agents, as well as dietary compounds, were proven to affect the apoptosis and proliferation in CRC. Most of them were tested in in vitro models. The clinical significance of their additional actions needs to be confirmed. To check the clinical value of such agents, prospective, controlled clinical studies are required.

2. Different molecular pathways of CRC development may determine the heterogeneous potential of any considered intervention and should be taken into account in the relevant study settings.

3. It seems conceivable that some drugs and dietary compounds may turn out to be valuable adjuncts in CRC prevention and therapy.

\section{The authors declare no conflict of interest.}

\section{References}

1. Fearon ER, Vogelstein B. A genetic model for colorectal tumorigenesis. Cell 1990; 61: 759-67.

2. Leggett $B$, Whitehall V. Role of the serrated pathway in colorectal cancer pathogenesis. Gastroenterology 2010; 138: 2088-100.

3. Pan MH, Lai CS, Wu JC, Ho CT. Molecular mechanisms for chemoprevention of colorectal cancer by natural dietary compounds. Mol Nutr Food Res 2011; 55: 32-45.

4. Nakamura T, Nomura S, Sakai T, Nariya S. Expression of bcl-2 oncoprotein in gastrointestinal and uterine carcinomas and their premalignant lesions. Hum Pathol 1997; 28: 309-15.

5. Palazzo JP, Kafka NJ, Grasso L, Chakrani F, Hanau C, Cuesta KH, Mercer WE. The role of p53, p21 WAF1/C1PI, and bcl-2 in radioresistant colorectal carcinoma. Hum Pathol 1997; 28: 1189-95.
6. Sinicrope FA, Hart J, Hsu HA, Lemoine M, Michelassi F, Stephens LC. Apoptotic and mitotic indices predict survival rates in lymph node-negative colon carcinomas. Clin Cancer Res 1999; 5: 1793 804.

7. Parsons DW, Wang TL, Samuels Y, et al. Colorectal cancer: mutations in a signalling pathway. Nature 2005; 436: 792.

8. Watson AJ, Merritt AJ, Jones LS, et al. Evidence of reciprocity of bcl-2 and p53 expression in human colorectal adenomas and carcinomas. Br J Cancer 1996; 73: 889-95.

9. Lipkin M, Reddy B, Newmark H, Lamprecht SA. Dietary factors in human colorectal cancer. Annu Rev Nutr 1999; 19: 545-86.

10. Baron J, Cole BF, Sandler RS, et al. A randomized trial of aspirin to prevent colorectal adenomas. N Engl J Med 2003; 348: 891-9.

11. Steinbach G, Lynch PM, Phillips RK, et al. The effect of celecoxib, a cyclooxygenase-2 inhibitor, in familial adenomatous polyposis. N Engl J Med 2000; 342: 1946-52.

12. Labayle D, Fischer D, Vielh P, et al. Sulindac causes regression of rectal polyps in familial adenomatous polyposis. Gastroenterology 1991; 101: 635-9.

13. Marszałek A, Szylberg L, Winiewska E, Janiczek M. Impact of COX-2, IL-1 $\beta$, TNF- $\alpha, I L-4$ and IL-10 on the process of carcinogenesis in the large bowel. Pol J Pathol 2012; 63: 221-7.

14. Hanif R, Pittas A, Feng Y, Koutsos MI, Qiao L, Staiano-Coico L, ShiffSI, Rigas B. Effects of non-steroidal anti-inflammatory drugs on proliferation and on induction of apoptosis in colon cancer cells by a prostaglandin-independent pathway. Biochem Pharmacol 1996; 52: 237-45.

15. Tsujii M, DuBois RN. Alterations in cellular adhesion and apoptosis in epithelial cells overexpressing prostaglandin endoperoxide synthase 2. Cell 1995; 83: 493-501.

16. Reinacher-Schick A, Seidensticker F, Petrasch S, Reiser M, Philippou S, Theegarten D, Freitag G, Schmiegel W. Mesalazine changes apoptosis and proliferation in normal mucosa of patients with sporadic polyps of the large bowel. Endoscopy 2000; 32: 245-54.

17. Bus PJ, Nagtegaal ID, Verspagct HW, Lamers CB, Geldof H, Van Krieken JH, Griffioen G. Mesalazine-induced apoptosis of colorectal cancer; on the verge of a new chemopreventive era. Aliment Pharmacol Ther 1999; 13: 1397-402.

18. Reinacher-Schick A, Schoeneck A, Graeven U, Schwarte-Waldhoff I, Schmiegel W. Mesalazine causes a mitotic arrest and induces caspase-dependent apoptosis in colon carcinoma cells. Carcinogenesis 2003; 24: 443-51.

19. Shureigi I, Chen D, Lee JJ, et al. 15-LOX-1: a novel molecular target of nonsteroidal anti-inflammatory drug-induced apoptosis in colorectal cancer cells. J Natl Cancer Inst 2000; 92: 1136-42.

20. Hawcroft G, D’Amico M, Albanese C, Markham AF, Pestell RG, Hull $M A$. Indomethacin induces differential expression of beta-catenin, gamma-catenin and T-cell factor target genes in human colorectal cancer cells. Carcinogenesis 2002; 23: 107-14.

21. Li B, Cao F, Zhu Q, Li B, Gan M, Wang D. Perioperative cimetidine administration improves systematic immune response and tumor infiltrating lymphocytes in patients with colorectal cancer. Hepatogastroenterology 2013; 60: 244-7.

22. Deva S, Jameson M. Histamine type 2 receptor antagonists as adjuvant treatment for resected colorectal cancer. Cochrane Database Syst Rev 2012; 8: CD007814.

23. Lin CY, Bai DJ, Yuan HY, Wang K, Yang GL, Hu MB, Wu ZQ, Li Y. Perioperative cimetidine administration promotes peripheral blood lymphocytes and tumor infiltrating lymphocytes in patients with gastrointestinal cancer: Results of a randomized controlled clinical trial. World J Gastroenterol 2004; 10: 136-42.

24. Wachtershauser A, Akoglu B, Stein J. HMG-CoA reductase inhibitor mevastatin enhances the growth inhibitory effect of butyrate in the colorectal carcinoma cell line Caco-2. Carcinogenesis 2001; 22: 1061-67.

25. Agarwal B, Rao CV, Bhendwal S, Ramey WR, Shirin H, Reddy BS, Holt PR. Lovastatin augments sulindac-induced apoptosis in colon cancer cells and potentiate chemopreventive effects of sulindac. Gastroenterology 1999; 117: 838-47.

26. Załęski A, Banaszkiewicz A, Walkowiak J. Butyric acid in irritable bowel syndrome. Prz Gastroenterol 2013; 8: 350-3. 
27. Wenzel U, Nickel A, Daniel H. Increased carnitine-dependent fatty acid uptake into mitochondria of human colon cancer cells induces apoptosis. J Nutr 2005; 135: 1510-4.

28. World Cancer Research Fund/American Institute for Cancer Research. Food, nutrition, physical activity and the prevention of cancer: a global perspective. AICR 2007.

29. Bingham SA, Day NE, Luben R, et al.; European Prospective Investigation into Cancer and Nutrition. Dietary fibre in food and protection against colorectal cancer in The European Prospective Investigation into Cancer and Nutrition (EPIC): an observational study. Lancet 2003; 361: 1496-501.

30. Aune D, Chan DS, Lau R, Vieira R, Greenwood DC, Kampman E, Norat T. Dietary fibre, whole grains, and risk of colorectal cancer: systematic review and dose-response meta-analysis of prospective studies. BMJ 2011; 343: d6617.

31. Burns AJ, Rowland IR. Anti-carcinogenicity of probiotics and prebiotics. Curr Issues Intest Microbiol 2000; 1: 13-24.

32. Zhong L, Zhang X, Covasa M. Emerging roles of lactic acid bacteria in protection against colorectal cancer. World J Gastroenterol 2014; 20: 7878-86.

33. Bernstein C, Bernstein H, Garewal H, et al. A bile acid-induced apoptosis assay for colon cancer risk and associated quality control studies. Cancer Res 1999; 59: 2353-7.

34. Goel A, Aggarwal BB. Curcumin, the golden spice from Indian saffron, is a chemosensitizer and radiosensitizer for tumors and chemoprotector and radioprotector for normal organs. Nutr Cancer 2010; 62: 919-30.

\section{Address for correspondence}

\section{Miroslaw Kiedrowski}

Faculty of Human Nutrition and Consumer Sciences

Warsaw University of Life Sciences - SGGW

Nowoursynowska 159 C

02-776 Warsaw, Poland

e-mail: mkiedrow@mp.pl

Submitted: 3.07.2014

Accepted: 23.07 .2014 